\title{
The Equivalence Principle and the bending of light
}

\author{
Rafael Ferraro* \\ Instituto de Astronomía y Física del Espacio, \\ Casilla de Correo 67, Sucursal 28, 1428 Buenos Aires, Argentina \\ Departamento de Física, Facultad de Ciencias Exactas y Naturales, \\ Universidad de Buenos Aires, Ciudad Universitaria, Pabellón I, \\ 1428 Buenos Aires, Argentina
}

\begin{abstract}
The apparent discrepancy between the bending of light predicted by the equivalence principle and its corresponding value in general relativity is resolved by evaluating the deflection of light with respect to a direction that is parallel transported along the ray trajectory in 3-space. In this way the bending predicted by the equivalence principle is fulfilled in general relativity and other alternative metric theories of gravity.
\end{abstract}

\section{Introduction}

In 1911, five years before general relativity was formulated, Einstein used the equivalence principle to calculate the bending of a light ray in a weak gravitational field [1]. By considering the Doppler shift suffered by a light ray in a reference system that moves with acceleration $\mathbf{a}=-\mathbf{g}$ in the absence of a gravitational field, Einstein deduced that the frequency of light must experience a shift $\nu(0)=\nu(H)\left(1+g H / c^{2}\right)$ when the light moves a height $H$ in a weak static gravitational field $\mathbf{g}\left(g H / c^{2}<<1\right)$. Because Einstein did not yet possess a metric theory of gravity, he reasoned that the gravitational redshift compels us to use "clocks of unlike constitution for measuring time at places with differing gravitational potential" [1]. In fact, because the number of wave maxima per unit of time should not vary at different heights in a static gravitational field, a clock must go $\left(1+g H / c^{2}\right)$ times more slowly at $z=H$ than at $z=0$. Einstein concluded that the velocity of light is $c$ when it is measured with clocks of equal constitution; instead, the use of clocks of unequal constitution implies that the velocity of propagation should increase at the same rate

*ferraro@iafe.uba.ar 
as the clocks go slower: $c(H)=c(0)\left(1+g H / c^{2}\right)$. ("The principle of constancy of the velocity of light holds good according to this theory in a different form from that which usually underlies the ordinary theory of relativity" [1]). Thus a plane wave traveling transversely to a gravitational field changes its direction of propagation -literally, it falls- because the secondary fronts of the Huygens' construction advance faster where the gravitational potential is higher. In fact, the deflection $\phi$ in Fig. 1 is

$$
\phi \simeq \frac{\left[c_{2}-c_{1}\right] \Delta t}{H} \simeq \frac{g H / c^{2}}{H} c \Delta t \simeq g c^{-2} \Delta x .
$$

Remarkably, the result in Eq. (11) can also be obtained by a direct application of the equivalence principle to the trajectory of a particle traveling at the speed of light. In the accelerated system $(\mathbf{a}=-g \hat{\mathbf{z}})$ such a particle follows the equations of motion $x \simeq c t$ and $z=-\frac{1}{2} g t^{2}$. Therefore, its trajectory is $z \simeq-\frac{1}{2} g c^{-2} x^{2}$, so its slope after a displacement $\Delta x$ is $d z / d x \simeq-g c^{-2} \Delta x$ in agreement with Eq. (11).

It is frequently emphasized that Eq. (1) does not agree with the experimentally tested bending of light predicted by general relativity 2, but only equals half this value (see for instance Refs. [3] and [4]). This issue has deserved much attention, and it is usually said that the equivalence principle contributes one half to the deflection while the other half comes from the spatial curvature [5]. In fact, the equivalence principle only prescribes the form $g_{00}=1+2 \Phi c^{-2}$ for the temporal part of the metric associated with a weak gravitational potential $\Phi(\mathbf{r})$, but says nothing about the spatial geometry, which certainly influences a light ray. However, this way of relating the equivalence principle to the relativistic bending of light diminishes the value of the equivalence principle by suggesting that the equivalence principle is unable to predict a quantitative physical result.

To reinstate the equivalence principle as a full statement about the local behavior of matter and radiation in a gravitational field so that it can yield results in quantitative agreement with those from a metric theory of gravity, we should first establish the kind of deflection that is referred to by the equivalence principle in the context of a metric theory of gravity (see Ref. [6] for an approach of this type). In general, the notion of deflection of a trajectory relative to a fixed direction entails the parallel transport of that direction along the trajectory. As it is well known, the angle between a trajectory and a parallel transported direction does not change when the trajectory is a geodesic. Instead, if the trajectory is not a geodesic, then the angle will change (the trajectory deflects). The world line of a ray light is a geodesic of the 4-dimensional spacetime. However, the equivalence principle for the bending of light does not refer to the world line, but contains a statement about the ray trajectory in 3 -space. As we will see, the trajectory in 3-space is not a geodesic. Thus the angle between the trajectory and a parallel transported direction changes along the trajectory. Therefore, the equivalence principle should be regarded as a statement about this angle, which is a geometric (invariant) object of 3 -space.

Our first task will be to determinate the trajectory of a light ray in the curved 3-space, together with its affine parameter (that is, the length of the 
trajectory). Then we will calculate the parallel transport of a vector along the trajectory of the light. Finally we will show that a light ray deflects at the angle predicted by the equivalence principle.

\section{The trajectory of a light ray}

We will work in a weak gravitational field geometry described by the static metric

$$
d s^{2}=\left(1+2 \Phi(\mathbf{r}) c^{-2}\right) c^{2} d t^{2}-\left(1-2 \gamma \Phi(\mathbf{r}) c^{-2}\right)\left(d x^{2}+d y^{2}+d z^{2}\right),
$$

where the 3 -space metric includes the post-newtonian parameter $\gamma$ that is equal to 1 in general relativity [4, 8]. We can use $\gamma$ to track the spatial geometry contribution to the bending of light.

The behavior of a light ray is obtained from the equation for null geodesics. Equivalently, we can start from the first integral

$$
g^{i j} p_{i} p_{j}=0, \quad p_{i} \equiv g_{i k} \frac{d x^{k}}{d \lambda},
$$

where $\lambda$ is an affine parameter. Let us consider a ray satisfying the initial conditions $d y / d \lambda=0$ and $d z / d \lambda=0$. For simplicity, we will consider a region where the gravitational field is nearly uniform: $\Phi=g z$, so $p_{0}, p_{x}$, and $p_{y}$ are conserved magnitudes. Initially (at $x=0, z=0$ ) Eq. (3) reads

$$
p_{0}^{2}-p_{x}^{2}=0 .
$$

Because $p_{0}$ and $p_{x}$ are conserved, Eq. (何) implies that $p_{0}^{2}=p_{x}^{2}$ for all value of $z$. Therefore, Eq. (3) becomes

$$
\left(1+2 g z c^{-2}\right)^{-1} p_{x}^{2}-\left(1-2 \gamma g z c^{-2}\right)^{-1} p_{x}^{2}-\left(1-2 \gamma g z c^{-2}\right)\left(\frac{d z}{d \lambda}\right)^{2}=0 .
$$

If we retain only terms to first-order in $g z c^{-2}$, we have

$$
-2(1+\gamma) g z c^{-2} p_{x}^{2} \simeq\left(\frac{d z}{d \lambda}\right)^{2}
$$

and

$$
z=-\frac{1+\gamma}{2} p_{x}^{2} g c^{-2} \lambda^{2}+O\left(\lambda^{4}\right)
$$

Because

$$
\frac{d x}{d \lambda}=g^{x x} p_{x}=-\left(1-2 \gamma g z c^{-2}\right)^{-1} p_{x}
$$

implies that

$$
x=-p_{x} \lambda+O\left(\lambda^{3}\right),
$$


we have

$$
z(x)=-\frac{1+\gamma}{2} g c^{-2} x^{2}+O\left(x^{4}\right) .
$$

After a displacement $\Delta x$, the slope of the trajectory $z(x)$ is

$$
\frac{d z}{d x} \simeq-(1+\gamma) g c^{-2} \Delta x .
$$

This result clearly separates the equivalence principle contribution due to $g_{00}$ from the one associated with the spatial geometry which is proportional to $\gamma$. Both contributions are equal in general relativity.

\section{Bending of light in 3-space}

Any trajectory in the 3 -space can be characterized by its tangent 3-vector $\bar{U}$ whose components are $\bar{U}^{\alpha}=d x^{\alpha} / d l(\alpha=1,2,3)$; the affine parameter $l$ is the length of the trajectory. In the 3 -space of the static metric (2), the infinitesimal length of the trajectory (10) is

$$
d l^{2}=\left(1-2 \gamma \Phi c^{-2}\right)\left(d x^{2}+d z^{2}\right)=\left(1-2 \gamma g c^{-2} z(x)\right)\left[1+(d z / d x)^{2}\right] d x^{2} .
$$

Thus, $d l$ is

$$
d l=\left[1+\frac{1}{2}(1+2 \gamma)(1+\gamma) g^{2} c^{-4} x^{2}+O\left(x^{4}\right)\right] d x,
$$

and the 3 -vector $\bar{U}$ that is tangent to the ray trajectory is

$$
\begin{aligned}
\bar{U}^{x} & =\frac{d x}{d l}=1-\frac{1}{2}(1+2 \gamma)(1+\gamma) g^{2} c^{-4} x^{2}+O\left(x^{4}\right) \\
\bar{U}^{y} & =\frac{d y}{d l}=0 \\
\bar{U}^{z} & =\frac{d z}{d l}=\frac{d z}{d x} \frac{d x}{d l}=-(1+\gamma) g c^{-2} x+O\left(x^{3}\right) .
\end{aligned}
$$

We can easily verify that the covariant derivative $D \bar{U} / D l$ does not vanish, so the trajectory of the light ray is not a geodesic in 3 -space.

As mentioned in Sec. 1, the bending of light is measured by the angle between the trajectory and a direction that is parallel transported along it. So we

are going to parallel transport the 3 -vector $\bar{V}=\left(\bar{V}^{x}, 0, \bar{V}^{z}\right)$ along the 3 -curve described by the tangent vector $\bar{U}$. This parallel transport is defined by the equation

$$
\left(\frac{D \bar{V}}{D l}\right)^{\alpha} \equiv \frac{d \bar{V}^{\alpha}}{d l}+\bar{\Gamma}_{\beta \delta}^{\alpha} \bar{U}^{\beta} \bar{V}^{\delta}=0,
$$

where $\bar{\Gamma}_{\beta \delta}^{\alpha}$ are the Christoffel symbols associated with the 3 -space geometry. The only non-zero Christoffel symbols in which we are interested are

$$
\bar{\Gamma}_{x z}^{x}=\bar{\Gamma}_{z x}^{x}=-\gamma g c^{-2}, \bar{\Gamma}_{x x}^{z}=\gamma g c^{-2}, \bar{\Gamma}_{z z}^{z}=-\gamma g c^{-2} .
$$


Let us choose $\bar{V}(0)=\bar{U}(0)=(1,0,0)$ as the initial condition at $x=0, z=0$. Thus the solution of Eq. (15) is

$$
\begin{aligned}
\bar{V}^{x} & =1-\frac{1}{2}(1+2 \gamma) \gamma g^{2} c^{-4} x^{2}+O\left(x^{4}\right) \\
\bar{V}^{z} & =-\gamma g c^{-2} x+O\left(x^{3}\right) .
\end{aligned}
$$

The angle between the directions of $\bar{V}$ and the trajectory is

$$
\begin{aligned}
\cos \phi(x)= & \frac{\bar{U} \cdot \bar{V}}{|\bar{U}||\bar{V}|}=\frac{\bar{g}_{\alpha \beta} U^{\alpha} V^{\beta}}{\left[\bar{g}_{\alpha \beta} U^{\alpha} U^{\beta}\right]^{1 / 2}} \\
& =\left(1-2 \gamma g z(x) c^{-2}\right)^{1 / 2}\left(\bar{U}^{x} \bar{V}^{x}+\bar{U}^{z} \bar{V}^{z}\right)\left(\bar{U}^{x} \bar{U}^{x}+\bar{U}^{z} \bar{U}^{z}\right)^{-1 / 2}(1,8)
\end{aligned}
$$

where we utilized the fact that $|\bar{V}|=1$ is preserved by the parallel transport in 3 -space. Therefore

$$
\cos \phi(x)=1-\frac{1}{2} g^{2} c^{-4} x^{2}+O\left(x^{4}\right),
$$

so the deflection angle after a small displacement $\Delta x$ is

$$
\phi \simeq g c^{-2} \Delta x,
$$

in agreement with the result (11) obtained by using the equivalence principle.

\section{Conclusion}

The use of the equivalence principle can satisfactorily account for the bending of light in the context of the weak gravitational field metric (2). The result from the equivalence principle corresponds to a physically meaningful quantity: it is the angle formed by the trajectory of the light ray and a direction that is parallel transported along that trajectory in 3-space. The post-newtonian parameter $\gamma$ does not enter the deflection angle, although it obviously affects both the trajectory and the parallel transport. In this way, any metric theory of gravity that leads to the weak field behavior of Eq. (2) contains the bending of light predicted by the equivalence principle.

\section{Acknowledgments}

This work was supported by Universidad de Buenos Aires (Proy. X143) and Consejo Nacional de Investigaciones Científicas y Técnicas (Argentina). 


\section{References}

[1] A. Einstein, Über den Einflußder Schwerkraft auf die Ousbreitung des Lichtes, Annalen der Physik 35, 898-908. (1911). Translated in A. Einstein, H. A. Lorentz, H. Minkowski, and H. Weyl, The Principle of Relativity: a Collection of Original Memoirs (Dover, NY, 1958).

[2] F. W. Dyson, A. S. E. Eddington, and C. R. Davidson, "A determination of the deflection of light by the sun's gravitational field, from observations made at the total eclipse of May 29, 1919," Phil. Trans. Roy. Soc. A 220, 291-333 (1920); D. S. Robertson, W. E. Carter, and W. H. Dillinger, "New measurement of solar gravitational deflection of radio signals using VLBI," Nature 349, 768-770 (1991).

[3] C. Lanczos, The Variational Principles of Mechanics (Dover, NY, 1986).

[4] S. Weinberg, Gravitation and Cosmology: Principles and Applications of the General Theory of Relativity (Wiley, NY, 1972).

[5] L. Parker, "Motion in Schwarzschild field I. Precession of a moving gyroscope," Am. J. Phys. 37 309-312 (1969); ibid., "Motion in Schwarzschild field II. Deflection of light," Am. J. Phys. 37, 313-314 (1969).

[6] R. P. Comer and J. D. Lathrop, "Principle of equivalence and the deflection of light by the sun," Am. J. Phys. 46, 801-803 (1978).

[7] C. Misner, K. Thorne, and J. A. Wheeler, Gravitation (Freeman, San Francisco, 1973).

[8] C. M. Will, Theory and Experiment in Gravitational Physics (Cambridge University Press, Cambridge, 1993). 
Figure 1: Huygens construction of the wave front in a static gravitational field. 